EPJ Web of Conferences 47, 14001 (2013)

DOI: $10.1051 /$ epjconf/20134714001

(C) Owned by the authors, published by EDP Sciences, 2013

\title{
Outflows and disks of brown dwarfs with SMA, CARMA and ALMA
}

\author{
Ngoc Phan-Bao ${ }^{1,2, a}$, Chin-Fei Lee ${ }^{2}$, Paul Ho ${ }^{2,3}$, Eduardo Martín ${ }^{4}$ \\ and Do D. Tho ${ }^{1}$ \\ ${ }^{1}$ Department of Physics, HCMIU, Vietnam National University, Block 6, Linh Trung Ward, \\ Thu Duc District, HCM, Vietnam \\ ${ }^{2}$ Institute of Astronomy and Astrophysics, Academia Sinica, PO Box 23-141, \\ Taipei 106, Taiwan \\ ${ }^{3}$ Harvard-Smithsonian Center for Astrophysics, Cambridge, MA 02138, USA \\ ${ }^{4}$ Centro de Astrobiologia (CAB-CSIC), Ctra. Ajalvir km 4, 28850 Torrejón de Ardoz, \\ Madrid, Spain
}

\begin{abstract}
Brown dwarfs are on the dividing line between planets and stars. Up to date, about 1,000 brown dwarfs, including the coolest known brown dwarfs with temperatures of $\sim 300 \mathrm{~K}$ as cool as the human body, have been discovered. However, the origin of these objects is still not well understood. Here we report our study of molecular outflows and disks of young very-low mass stars and brown dwarfs in $\rho$ Ophiuchi and Taurus using the Submillimeter Array (SMA) and the Combined Array for Research in Millimeterwave Astronomy (CARMA). The observations of brown dwarfs at early stages provide key information to understand their formation mechanism as well as planet formation around these very low-mass objects. We also discuss future observations of brown dwarfs with the Atacama Large Millimeter/submillimeter Array (ALMA)
\end{abstract}

\section{INTRODUCTION}

It is still not clear whether brown dwarfs $(\mathrm{BD})\left(13-75 M_{\mathrm{J}}\right)$ are formed in the same manner as low-mass stars or by different mechanisms. Two major models, which are able to produce large numbers of BDs (see [35] and references therein), have been proposed for the BD formation: (1) Starlike formation: BD mass cores are produced by turbulent fragmentation of molecular clouds by shock compression [22] or gravitational fragmentation [5], these pre-BD cores are dense enough to be gravitationally unstable. This mechanism is comparable to the formation of low-mass stars; (2) Ejection scenario: BDs are simply very low-mass protostellar embryos that are ejected from unstable multiple protostellar systems by the dynamical interaction of embryos $[4,27]$.

Observations of the statistical properties of young brown dwarfs in different star-forming regions such as the initial mass function, velocity dispersion, multiplicity, accretion disks and jets (see [15] and references therein) have demonstrated that all these properties of BDs appear to form a continuum with those of low-mass stars. The first detections of bipolar molecular outflows from young BDs [23, 24] have indicated that the molecular outflow process occurs in BDs as a scaled-down version of that seen in low-mass stars. All these observations support the scenario that BDs form as low-mass stars do.

\footnotetext{
ae-mail: pbngoc@hcmiu.edu.vn
}

This is an Open Access article distributed under the terms of the Creative Commons Attribution License 2.0, which permits unrestricted use, distribution, and reproduction in any medium, provided the original work is properly cited. 
However, all the BDs observed so far are class II/III objects, which are in the last stages of BD formation. These objects are reaching their final masses and will end up with BD masses. Brown dwarfs at ealier stages, such as class I and 0 as well as BD cores predicted by starlike formation models (e.g., turbulent/gravitational fragmentation) have not been observationally confirmed yet. Therefore, the BD formation process is still not understood. One should note that detections of molecular outflows from class I/0 proto-BD candidates have been reported (L1048-IRS, [6]; L1148-IRS, [26]), however, the estimated masses of the central objects are uncertain and it is not known whether these candidates end up in BDs or low-mass stars. Recently, the first identification of a confirmed pre-BD, Oph B-11 in $\rho$ Ophiuchi, has been reported [3]. The BD core is a self-gravitating condensation of gas and dust with a mass of $0.02-0.03 M_{\odot}$ and the estimated final mass of the core in the range of $0.01-0.04 M_{\odot}$ is also substellar. This provides the first test of starlike models for BD formation. It is therefore important to identify and study more BDs at the earliest stages to complete our understanding of their formation mechanism [25].

Here we report the update of our search and characterization of molecular outflows from young BDs using SMA and CARMA. The observed targets consist of 7 brown dwarfs and 1 very low-mass star in two nearby star-forming regions $\rho$ Ophiuchi and Taurus.

\section{SAMPLE SELECTION}

We selected 2 BDs in $\rho$ Ophiuchi, 5 BDs and 1 very low-mass star in Taurus (see Table 1). These targets have masses ranging from 30 to $90 \mathrm{M}_{\mathrm{J}}$. All these objects are strong accretors [14, 19-21]. Some of them (ISO-Oph 102, MHO 5, ISO-Oph 32, 2M $0438+2611$ ) show forbidden emission lines that could be associated with outflow activities. The $\mathrm{H} \alpha$ accretion emission profiles of ISO-Oph 102, $2 \mathrm{M} 0414+2811$ and GM Tau particularly show an extreme P Cygni profile [20, 21, 34] with a dip on the blueward side, indicating a mass loss process analogous to that seen in higher mass $\mathrm{T}$ Tauri stars. These objects are therefore good targets for our molecular outflow search.

\section{OBSERVATIONS AND DATA REDUCTION}

\subsection{SMA}

We have observed three BDs ISO-Oph 102, ISO-Oph 32 and GM Tau and the very low-mass star (MHO 5) with the receiver band at $230 \mathrm{GHz}$ of the $\mathrm{SMA}^{1}$ [9]. The $2 \mathrm{GHz}$ - and $4 \mathrm{GHz}$-wide sidebands have been used. The SMA correlator was configured with high spectral resolution bands of 512 channels per chunk of $104 \mathrm{MHz}$ for ${ }^{12} \mathrm{CO},{ }^{13} \mathrm{CO}$, and $\mathrm{C}^{18} \mathrm{O} J=2 \rightarrow 1$ lines, giving a channel spacing of $0.27 \mathrm{kms}^{-1}$. A lower resolution of $3.25 \mathrm{MHz}$ per channel was set up for the remainder of each sideband. Strong quasars have been observed for gain (e.g., $1625-254,0428+329,3 \mathrm{C} 111$ ) and passband (e.g., 3C 279, 3C 454.3, 3C 273) calibration. Uranus was used for flux calibration. The data were calibrated using the MIR software package and further analysis was carried out with the MIRIAD package adapted for the SMA. The compact configuration was used, resulting a synthesized beam of about 3 ". $6 \times 2$ ". 8 . The rms sensitivity was about $1 \mathrm{mJy}$ for the continuum, using both sidebands and $\sim 0.2 \mathrm{Jy} \mathrm{beam}^{-1}$ per channel for the line data. The primary FWHM beam is about $50^{\prime \prime}$ at the observed frequencies.

\subsection{CARMA}

Four BDs in Taurus 2M $0441+2534,2 \mathrm{M} 0439+2544,2 \mathrm{M} 0438+2611$ and $2 \mathrm{M} 0414+2811$ were observed with CARMA at $230 \mathrm{GHz}$ for ${ }^{12} \mathrm{COJ}=2 \rightarrow 1$. All $500 \mathrm{MHz}$-wide bands, which may be

\footnotetext{
1 The Submillimeter Array is a joint project between the Smithsonian Astrophysical Observatory and the Academia Sinica Institute of Astronomy and Astrophysics and is funded by the Smithsonian Institution and the Academia Sinica.
} 


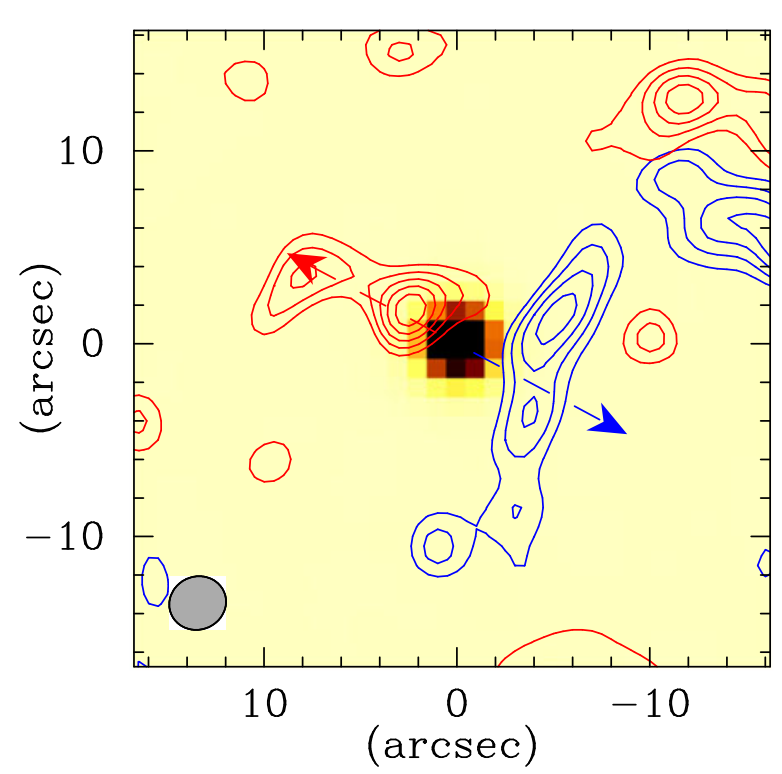

Figure 1. An overlay of the J-band (1.25 $\mu \mathrm{m})$ near-infrared Two Micron All Sky Survey (2MASS) image of MHO 5 and the integrated intensity in the carbon monoxide (CO $J=2-1)$ line emission from 2.45 to $6.15 \mathrm{kms}^{-1}$ lineof-sight velocities. The blue and red contours represent the blueshifted (integrated over 2.45 and $3.77 \mathrm{kms}^{-1}$ ) and redshifted (integrated over 4.56 and $6.15 \mathrm{kms}^{-1}$ ) emissions, respectively. The contours are $4,6,8$,...times the rms of $0.12 \mathrm{Jy}_{\text {beam }}^{-1} \mathrm{~km} \mathrm{~s}^{-1}$. The very low-mass star (MHO 5, $90 M_{\mathrm{J}}$ ) is visible in the J-band image. The position angle of the outflow is about $62^{\circ}$. The outflow directions are indicated by the blue and red arrows. The blue- and redshifted gas lobes are displaced on opposite sides of the star center with an offset of about $2^{\prime \prime}$. An elongated structure is visibly seen in the blueshifted component while the redshifted one shows an extended component (see Sec. 4.3 for discussion). The gas lobes in the top right corner are expected to be outflows from neaby massive outflows in the L1551 molecular cloud (see Fig. 4 and Fig. 15 in [18]). The synthesized beam is shown in the bottom left corner.

positioned independently with the IF bandwith, were used for $\mathrm{CO}$ search with different spectral resolutions. These bands were configured with channel widths of $0.122 \mathrm{MHz}$ or $0.16 \mathrm{kms}^{-1}, 0.488 \mathrm{MHz}$ or $0.64 \mathrm{kms}^{-1}$, and $31.25 \mathrm{MHz}$ or $40.7 \mathrm{kms}^{-1}$. The quasars 3C 111 and 3C 84 have been observed for gain passband, and flux calibration. The data were calibrated using the MIRIAD package optimized for the CARMA. The D configuration was used, giving a synthesized beam of about $2.5^{\prime \prime} \times 2.0^{\prime \prime}$. The primary FWHM beam is about $36^{\prime \prime}$ at $230 \mathrm{GHz}$.

\section{RESULTS AND DISCUSSION}

\subsection{Bipolar molecular outflows}

We have detected bipolar molecular outflows from three sources: ISO-Oph 102 [23] in $\rho$ Ophiuchi, MHO 5 [24] and GM Tau in Taurus. Figure 1 presents an overlay of a near-infrared image of MHO 5 and the integrated intensity in the carbon monoxide $(\mathrm{CO} J=2-1)$ line emission. Two blue- and redshifted $\mathrm{CO}$ lobes are detected around the position of MHO 5. Both lobes show elongated structures and they are not symmetrically displaced on opposite sides of the MHO 5 position as was seen in ISOOph 102. However the position-velocity ( $\mathrm{P}-\mathrm{V})$ diagram for the $\mathrm{CO}$ emission cut at a position angle of $62^{\circ}$ clearly indicates two blue- and red-shifted components (Fig. 2). The elongated structures are probably due to a mixture of outflows from MHO 5 and nearby massive outflows from class 0 and I 


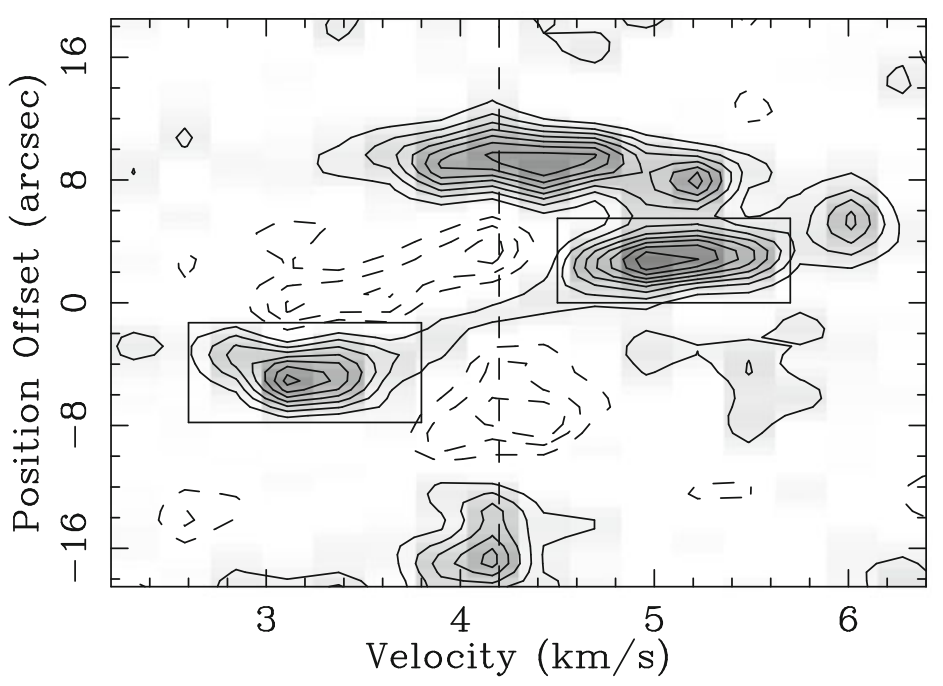

Figure 2. Position-Velocity (PV) cut diagram for $\mathrm{CO} J=2 \rightarrow 1$ emission at a position angle of $62^{\circ}$. The contours are $-8,-6,-4,-2,2,4,6,8$,..times the rms of $0.1 \mathrm{Jy}_{\text {beam }}{ }^{-1}$. The blueshifted $\left(2.6-3.8 \mathrm{~km} \mathrm{~s}^{-1}\right)$ and redshifted $\left(4.5-5.7 \mathrm{~km} \mathrm{~s}^{-1}\right)$ components expected from MHO 5 are indicated by boxes, which we take to estimate the MHO 5 outflow properties (see Sec. 4.1 for discussion). The gas velocity at the source position is $4.2 \pm 0.3 \mathrm{~km} \mathrm{~s}^{-1}$, which we take to be the systemic velocity of the very low-mass star as indicated by the dashed line.

Table 1. Young very low-mass objects in $\rho$ Ophiuchi and Taurus observed with SMA and CARMA.

\begin{tabular}{lccccccc}
\hline Target & Array & $\begin{array}{c}\text { Mass } \\
\left(M_{\mathrm{J}}\right)\end{array}$ & Region & $\begin{array}{c}\log \dot{M}_{\text {acc }} \\
\left(M_{\odot} \mathrm{yr}^{-1}\right)\end{array}$ & $\begin{array}{c}\log M_{\text {outflow }} \\
\left(M_{\odot}\right)\end{array}$ & $\begin{array}{c}\log \dot{M}_{\text {mass-loss }} \\
\left(M_{\odot} \mathrm{yr}^{-1}\right)\end{array}$ & References $^{\mathrm{a}}$ \\
\hline ISO-Oph 32 & SMA & 40 & $\rho$ Oph & -10.5 & - & - & {$[21]$} \\
ISO-Oph 102 & SMA & 60 & $\rho$ Oph & -9.0 & -3.8 & -8.9 & {$[21]$} \\
2M 0441 + 2534 & CARMA & 35 & Taurus & -11.3 & - & - & {$[20]$} \\
2M 0439+ 2544 & CARMA & 50 & Taurus & -11.3 & - & - & {$[20]$} \\
2M 0438 + 2611 & CARMA & 70 & Taurus & -10.8 & - & - & {$[20]$} \\
GM Tau & SMA & 73 & Taurus & -8.6 & -4.9 & -10.3 & {$[34]$} \\
2M 0414 + 2811 & CARMA & 75 & Taurus & -10.0 & - & - & {$[20]$} \\
MHO 5 & SMA & 90 & Taurus & -10.8 & -4.2 & -9.1 & {$[19]$} \\
\hline
\end{tabular}

${ }^{a}$ References for mass estimate, accretion rate.

protostars in the L1551 molecular cloud, a multigenerational star formation region [18]. We estimated the outflow properties of the three sources using the standard method $[2,7]$. The outflow masses and the mass-loss rates are given in Table 1. All these values are over 2 orders of magnitude smaller than the typical ones for $T$ Tauri stars [12, 13], demonstrating that the molecular outflow process in very low-mass objects is a scaled-down version of that in low-mass stars.

We have not detected CO molecular outflows from the remaining sources: ISO-Oph 32, 2M 0441+ $2534,2 \mathrm{M} 0439+2544,2 \mathrm{M} 0438+2611,2 \mathrm{M} 0414+2811$. There are two possibilities to explain the non-detections. First, their outflow emission might be too weak to be detectable. Second, these sources are class II objects, therefore the outflow process might have stopped. For the cases of ISO-Oph 32 and $2 \mathrm{M} 0414+2811$, the weak detection of optical jets in ISO-Oph 32 [33] and the P Cygni profile of $\mathrm{H} \alpha$ accretion emission observed in 2M $0414+2811$ [20] have implied outflow activities in these BDs. This therefore supports the first possibility that the molecular outflows from these sources are not detectable with the sensitivity of the current arrays. 


\section{Hot Planets and Cool Stars}

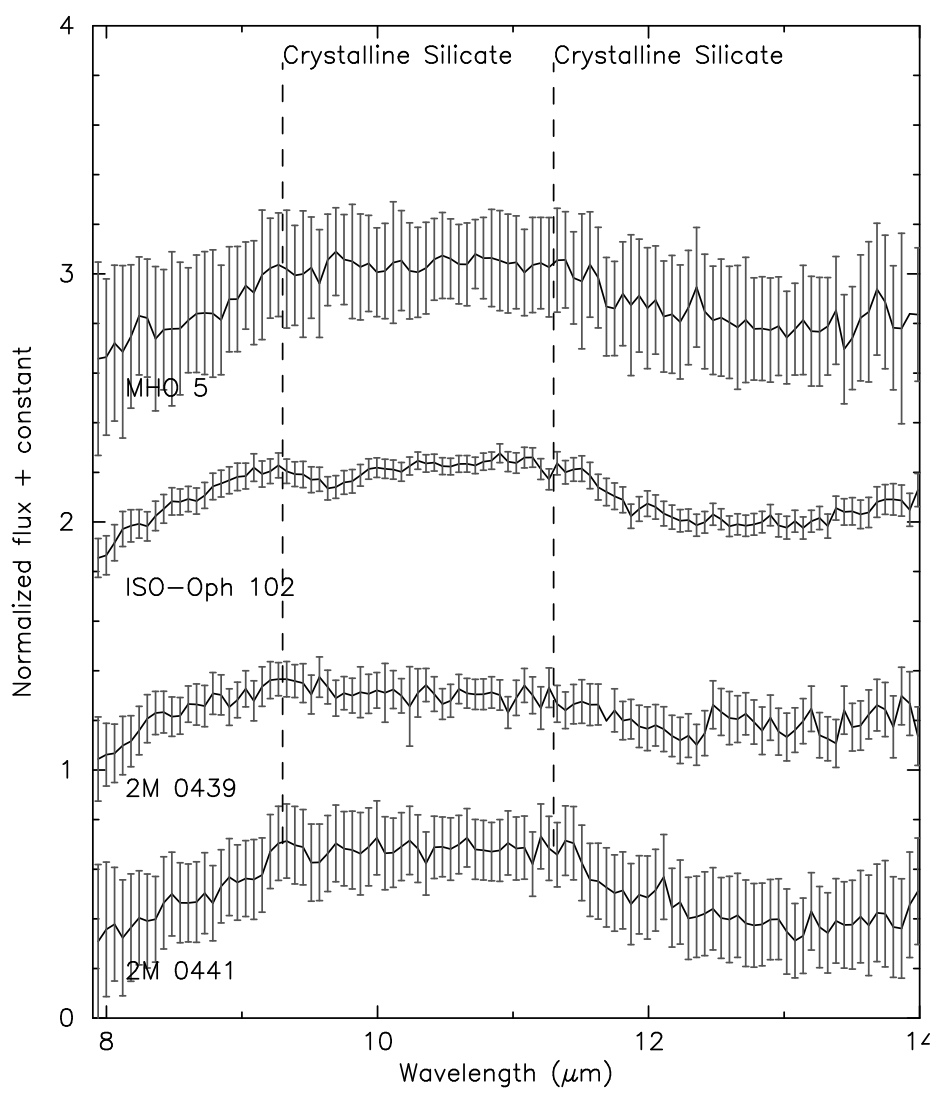

Figure 3. Spitzer infrared spectra of MHO 5, ISO-Oph 102, 2M 0439 and 2M 0441. Continuum subtraction was done following the literature $[1,32]$. The crystalline silicate features at $9.3 \mathrm{~mm}$ (mainly enstatite) and $11.3 \mathrm{~mm}$ (forsterite) are indicated.

\subsection{Brown dwarf disks}

A lot of work on disk modeling (e.g., $[10,16,28,31])$ has been done to estimate disk radii of young BDs. Scholz et al. [31] constructed spectral energy distributions (SED) to estimate disk radii of 20 young brown dwarfs in Taurus. They found that over $25 \%$ of the targets have disks with radii $>10 \mathrm{AU}$, this is significantly higher than the ratio of 5\% predicted by the ejection model [4]. The other authors have also found some brown dwarfs with disk radii $>20 \mathrm{AU}$. Using our SED constructed for ISO-Oph 102 [23], we estimated a disk radius of about $80 \pm 10$ AU for the BD. However, using ALMA Ricci et al. [30] successfully measured an upper limit of 40 AU on the disk radius of ISO-Oph 102, which is significantly smaller than our value. This suggests that disk radii of very low-mass objects derived from SEDs may be overestimated. More direct measurements of disk radii of young BDs are needed to confirm this discrepancy.

The detections of crystallization, grain growth and dust settling in the disks of many young brown dwarfs and very-low-mass stars have also been reported [1, 8, 17, 23, 24, 29]. All these detections have demonstrated that the planet formation process as seen in low-mass star disks also occurs in BD disks. Riaz et al. (2012) have presented a compositional analysis of silicate emission features of 20 brown dwarfs in Taurus. The authors have found that brown dwarfs exhibit stronger signs of dust processing in the cold component of the disk compared to T Tauri stars in the same region. They have also found that significant grain growth/dust settling has occurred at larger radii in the disk of some brown dwarfs, 


\section{EPJ Web of Conferences}

suggesting that dust processing mechanisms may not proceed simultaneously in the inner and outer regions for brown dwarf disks.

The IRS/Spitzer infrared (7.5-14.3 $\mu \mathrm{m})$ [11] spectra of objects (Figure 3) in our sample also show crystalline silicate features: enstatite $\left(\mathrm{MgSiO}_{3}\right)$ at $9.3 \mu \mathrm{m}$ and forsterite $\left(\mathrm{Mg}_{2} \mathrm{SiO}_{4}\right)$ at $11.3 \mu \mathrm{m}$. This provides additional evidences of grain growth and dust settling in the disks of these very low-mass objects. The coexistence of the molecular outflow and crystallization processes as detected in ISO-Oph 102, MHO 5 and GM Tau may favor the rocky planet formation around these objects as the outflows sweep away the gas and dust in the vicinity of the objects.

\subsection{Future observations with ALMA}

ALMA with 10-100 times more sensitive and 10-100 times better angular resolution than the current $\mathrm{mm} / \mathrm{submm}$ arrays is indeed an excellent instrument to detect molecular outflows from the least massive BDs in our sample (e.g., ISO-Oph 32), as well as to identify more BD cores (e.g., [3]) in star-forming regions. One should note that optical jets are not observable at very early phases of BD formation (e.g., BD cores, class 0), therefore the molecular outflow process plays a key role in identifying BDs at different stages of their formation, hence allowing us to understand the BD formation mechanism. ALMA also offers us excellent opportunities to directly measure disk radii and resolve the disk structure of BDs, which provide observational constraints on disk modeling in the substellar domain and key information on planet formation around BDs.

\section{SUMMARY}

We present here the properties of bipolar molecular outflows from two young BDs (ISO-Oph 102, GM Tau) and one very low-mass star (MHO 5). All these properties indicate that the outflow process in young very low-mass objects is very similar to that seen in young low-mass stars but scaled down by over two orders of magnitude. This strongly suggests that BDs and low-mass stars share the same formation mechanism and the terminal stellar/brown dwarf (even planetary) mass is not due to different formation mechanisms but more likely due to the initial mass of the cloud core.

N.P.-B has been supported by VietNam NAFOSTED grant 103.08-2010.07. N.P.-B would like to thank the organizers of the Hot Planets and Cool Stars conference. Support for CARMA construction was derived from the Gordon and Betty Moore Foundation, the Kenneth T. and Eileen L. Norris Foundation, the James S. McDonnell Foundation, the Associates of the California Institute of Technology, the University of Chicago, the states of California, Illinois, and Maryland, and the National Science Foundation. Ongoing CARMA development and operations are supported by the National Science Foundation under a cooperative agreement, and by the CARMA partner universities. This work is based in part on observations made with the Spitzer Space Telescope, which is operated by the Jet Propulsion Laboratory, California Institute of Technology, under a contract with NASA. This work has made use of the Centre de Données astronomiques de Strasbourg (CDS) database.

\section{References}

[1] D. Apai, et al., Science, 310, 834 (2005)

[2] P. André, J. Martín-Pintado, D. Despois, T. Montmerle, A\&A, 236, 180 (1990)

[3] André, P., et al., Science, 337, 69 (2012)

[4] M. Bate, et al., MNRAS, 332, 65 (2002)

[5] I.A. Bonnell, et al., MNRAS, 389, 1556 (2008)

[6] T.L. Bourke, A. Crapsi, P.C. Myers, et al., ApJ, 633, L129 (2005) 


\section{Hot Planets and Cool Stars}

[7] S. Cabrit, C. Bertout, ApJ, 348, 530 (1990)

[8] E. Furlan, et al., ApJS, 195, 3 (2011)

[9] P.T.P. Ho, J.M. Moran, K.Y. Lo, ApJ, 616, L1 (2004)

[10] P.M. Harvey, et al., ApJ, 744, L1 (2012)

[11] J.R. Houck, et al., ApJS, 154, 18 (2004)

[12] C.J. Lada, ARA\&A, 23, 267 (1985)

[13] R.M. Levreault, ApJS, 67, 283 (1988)

[14] K.L. Luhman, ApJ, 617, 1216 (2004)

[15] K.L. Luhman, V. Joergens, C. Lada, J. Muzerolle, I. Pascucci, R. White, in Protostars and Planets $V$, edited by B. Reipurth, D. Jewitt, \& K. Keil (Univ. Arizona Press, Tucson, 2007), p. 443

[16] K. Luhman, et al., ApJ, 666, 1219 (2007)

[17] B. Merín, et al., ApJ, 661, 361 (2007)

[18] G.H. Moriarty-Schieven, et al., ApJ, 645, 357 (2006)

[19] J. Muzerolle, L. Hillendbrand, N. Calvet, C. Briceño, L. Hartmann, ApJ, 592, 266 (2003)

[20] J. Muzerolle, K.L. Luhman, C. Briceño, L. Hartmann, N. Calvet, ApJ, 625, 906 (2005)

[21] A. Natta, L. Testi, J. Muzerolle, S. Randich, F. Comerón, P. Persi, A\&A, 424, 603 (2004)

[22] P. Padoan, \& A. Nordlund, ApJ, 617, 559 (2004)

[23] N. Phan-Bao, et al., ApJ, 689, L141 (2008)

[24] N. Phan-Bao, et al., ApJ, 735, 14 (2011a)

[25] N. Phan-Bao, C.-F. Lee, P. Ho, in New Technologies for Probing the Diversity of Brown Dwarfs and Exoplanets, 16, edited by E.L. Martín, J. Ge, \& W. Lin (EPJ Web of Conferences, 2011b), id.06003

[26] J. Kauffmann, et al., MNRAS, 416, 2341 (2011)

[27] B. Reipurth, C. Clarke, AJ, 122, 432 (2001)

[28] B. Riaz, et al., ApJ, 705, 1173 (2009)

[29] B. Riaz, et al., MNRAS, 420, 2603 (2012)

[30] L. Ricci, et al., ApJ, 761, L20 (2012)

[31] A. Scholz, R. Jayawardhana, K. Wood, ApJ, 645, 1498 (2006)

[32] R. van Boekel, et al., A\&A, 400, L21 (2003)

[33] E.T. Whelan, et al., ApJ, in press (arxiv:0910.2665), (2009)

[34] R.J. White, \& G. Basri, ApJ, 582, 1109 (2003)

[35] A. Whitworth, M.R. Bate, A. Nordlund, B. Reipurth, H. Zinnecker, in Protostars and Planets V, edited by B. Reipurth, D. Jewitt, \& K. Keil (Univ. Arizona Press, Tucson, 2007), p. 459 